Superalloys 2012: $12^{\text {th }}$ International Symposium on Superalloys

\title{
INTEGRATION AND AUTOMATION OF RESIDUAL STRESS AND SERVICE STRESS MODELING FOR SUPERALLOY COMPONENT DESIGN
}

\author{
Gangshu Shen, Nate Cooper, Nathan Ottow, Robert Goetz and John Matlik \\ Rolls-Royce Indianapolis; P.O. Box 420; Indianapolis, Indiana 46206
}

Keywords: Residual Stress Modeling, Residual Stress Integration, Residual Stress Automation, Disk Modeling, Disk Design

\begin{abstract}
The residual stress distribution generated during manufacturing processes of a jet engine component plays an important role in determining component life. The heat treatment, machining and pre-service spin variables (if used) contribute to the magnitude and location of residual stresses generated prior to service. Such variables have traditionally not been included in design optimization due to the lack of accurate and efficient process modeling design tools. Increased demand for higher speed and higher temperature disks has necessitated advanced multidisciplinary modeling tools in order to deliver high performance, robust and cost favorable solutions. Component process modeling that considers disk stresses through heat treatment, machining, pre-service spin and design operation has been linked and automated to minimize service stress for locations of interest. This paper uses a superalloy 718 disk as an example to illustrate integration and automation of residual stress and service stress modeling that will ultimately impact component life.
\end{abstract}

\section{Introduction}

The advent of Integrated Computational Materials Engineering (ICME) [1] and the need to drastically reduce design and make cycle times has placed greater importance on developing and demonstrating virtual/digital models. This will enable rapid and sustainable manufacture, critical to realizing potential time-tomarket savings. To successfully integrate design and manufacture, a method for integrating residual stresses arising from manufacture with in-service (or design mission) loading is required (see Figure 1).

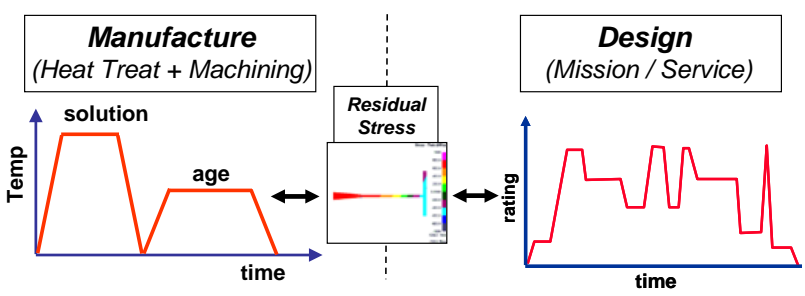

Figure 1. Schematic of integrating manufacturing residual stress with design mission / service loading.

Residual stresses are stresses that remain after the original loading and constraints have been removed from the part. Residual stresses in superalloy disk components originate from heat treatments and are produced when the material yields locally during post solution fast cooling (water quench, oil quench, air quench, etc.) [2-5]. The quenched-in residual stresses are then relaxed during aging, but not eliminated. Figure 2 shows a typical temperature-time curve for superalloy 718 disk heat treatment that involves solution and age. Residual stresses are reduced and redistributed further during machining from the 'as heat-treated' to a 'condition-of-supply' and then to the final component geometry. However, the residual stress inside the disk is still not negligible. It has an important impact on the life of the component during service.

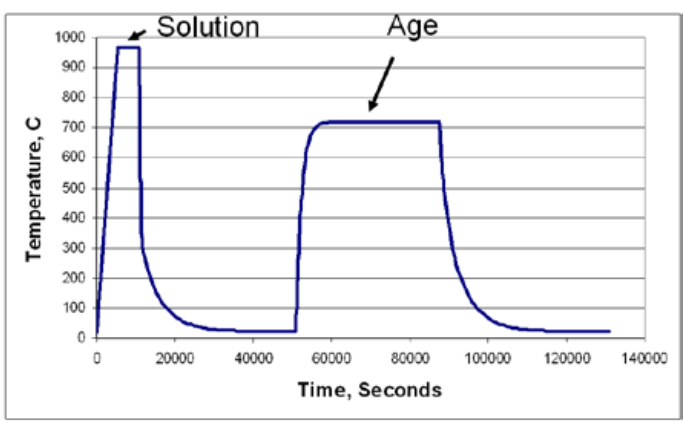

Figure 2. A typical temperature-time curve for superalloy 718 disk heat treatment that involves solution and age.

\section{Experiments and Model Validation}

Residual stress modeling simulates the heat treatment and machining processes using computer software such as DEFORM $^{\mathrm{TM}}$ [6] to predict the resultant residual stress in final components. Such modeling processes are becoming mature enough to correctly predict the residual stress of the final component [7]. Figure 3 shows the difference in absolute value in MPa between the prediction and mean measurement of the hoop stress for a portion of an RR1000 superalloy disk. The standard deviation of the difference between the prediction and measurement is around $50 \mathrm{MPa}$ (7 ksi).

\section{Hoop stress difference between prediction and mean measurement in} absolute value (MPa)

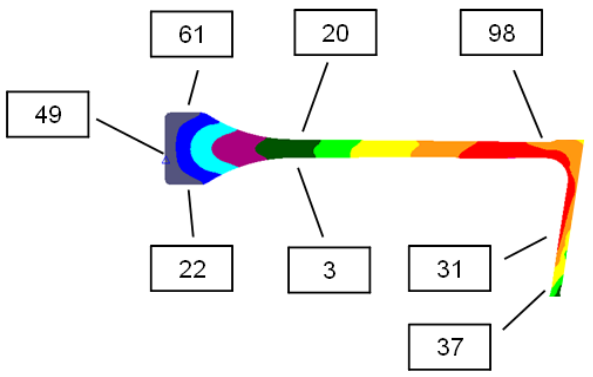

Figure 3. The difference in absolute value in MPa between the prediction and mean measurement of the hoop stress for a portion of a superalloy RR1000 disk 
With the improvement in residual stress prediction accuracy, the next step was to look at the relationship between cyclic spin life and predicted spin stress using residual stresses carried over from prior processing steps. Toward that end, two groups of $718 \mathrm{R} \& \mathrm{D}$ disks with documented spin test lives were investigated. The spin tests for the two groups of test article disks were run at room temperature and the spin hoop stress was the major contributor to failure. The two groups of disks had identical processing histories; the difference being the Group 1 disk was not pre-spun and Group 2 disk was pre-spun.

The modeling steps for the two groups of 718 disks (Figure 4) includes: a) solution heat treatment (Figure 2); b) age heat treatment (Figure 2); c) machining to component shape; d) prespin of disk (Group 2 only, 23,320 RPM); and e) spin at test speed (20,100 RPM). Group 1 disks failed at 7,963 cycles and Group 2 disks failed at 22,796 cycles. The reason for the longer life of the Group 2 disk was that pre-spinning yielded the bore, resulting in a compressive bore stress after pre-spinning and going into cyclic spin test (Figure 4d).

Figure 5 a) is a photo of the failed Group 1 disk. Failure analysis determined that the crack initiation location was at the bore; denoted by arrows. Figure 5 b) shows the predicted hoop stress during spinning, including residual stress. That is, the heat treated and machined disk was spun with the residual stresses as the initial condition rather a zero stress initial condition. Therefore, centrifugal stress is generated in a coupled manner rather than a simple, uncoupled superposition of residual and centrifugal stresses. The zone of the highest hoop stress during spinning for the Group 1 disk is located at the bore, which is the same crack initiation location indicated by failure analysis. The maximum and bore hoop stress are 1246 and 1164 MPa respectively.

The above stresses were used to compare with the Group 2 disk and the Trade Study disks to follow. Figure 6 a) is a photo of Group 2 disk that failed at 22,796 cycles. The possible crack initiation location is behind the filet. Figure $6 \mathrm{~b}$ ) shows the predicted spin hoop stress coupled with residual stress; the maximum spin hoop stress is located right behind the filet. The magnitude of the maximum spin hoop stress is $1193 \mathrm{MPa}$ and the spin hoop stress at the bore is $889 \mathrm{MPa}$. Both values are smaller than the Group 1 disk. The predicted locations and magnitudes of the maximum spin hoop stress for the two groups of 718 disks are consistent with the observed fracture pattern and relative life of the failed disks.
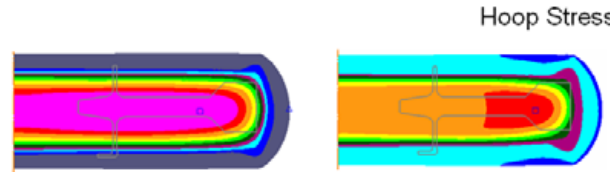

a) solution

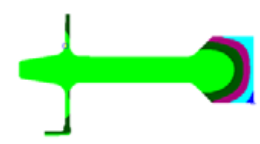

c) machining

b) age

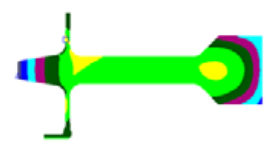

d) pre-spin (Group 2) Hoop Stress (MPa)

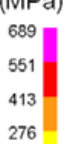

138 000

$-138$

$-551$

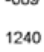

1240
1120
992
868
744
620
496
372
248
124
0.000

Figure 4 . The modeling steps for 718 disk include: a) solution heat treatment; b) age heat treatment; c) machining to component shape; d) pre-spin of disk (Group 2 only); and e) spin at temperature and speed.

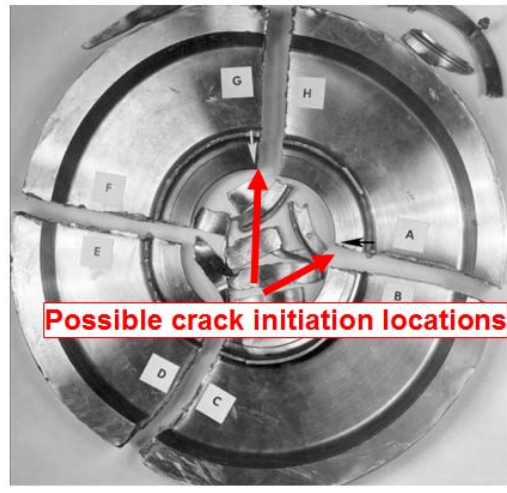

a)

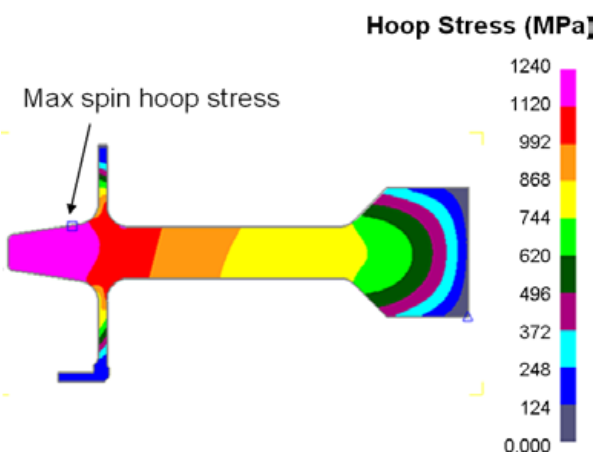

b)

Figure 5. a) Photo of Group 1 disk (7,963 cycles, arrow denotes crack initiation locations); b) predicted spin hoop stress with a maximum and bore stress of 1246 and 1164 MPa respectively. 


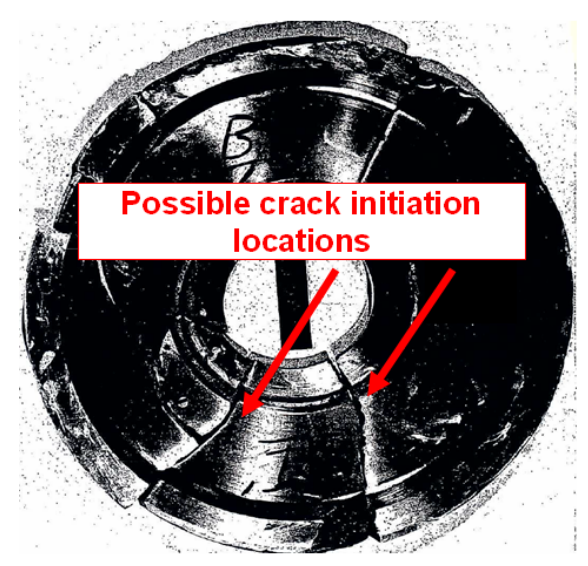

a)

Hoop Stress (MPa)

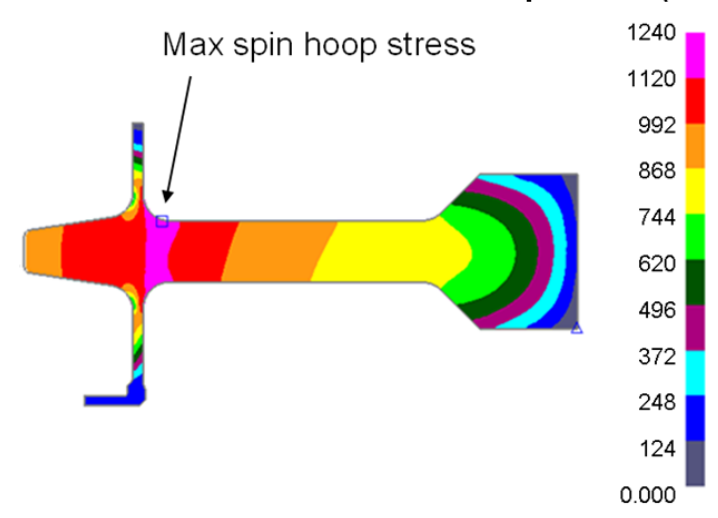

b)

Figure 6. a) Photo of Group 2 disk (22,796 cycles, the possible crack initiation location is behind the filet); b) The predicted spin hoop stress with a maximum of $1193 \mathrm{MPa}$ and a bore stress of $889 \mathrm{MPa}$

\section{Automation and Optimization}

An automated process modeling loop was developed to link all DEFORM modeling steps together, shortening the turn-around time for component design applications. Given the reasonable prediction of spin stress for a component with residual stress in the previous section, automation was a natural step toward an ICME approach to improve modeling efficiency. Figure 7 shows an example of such a loop that links: solution and aging heat treatment, machining, pre-spinning (if used), application of design point temperature and spin conditions, and the calculation of service stresses and life of the component.

A modeling Trade Study can be performed very efficiently with the automated process modeling loop shown in Figure 7. A Trade Study was investigated first, consisting of three conventional heat treatment scenarios coupled with pre-spinning. This was followed by a Design of Experiment (DOE) for quenching heat transfer

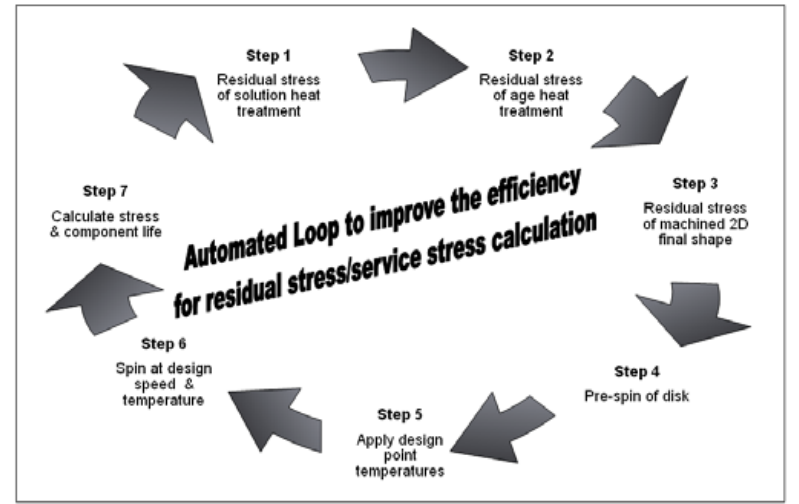

Figure 7. Automated process modeling loop developed to link all DEFORM modeling steps together.

coefficients. The Trade Study heat treatment conditions were; (1) a Pancake oil quench (Figure 8), which was the condition used for the Group 2 disk shown in Figure 4; (2) a Ring water quench (Figure 9), which was the $1^{\text {st }}$ Trade Study; and (3) a Ring oil quench (Figure 10), which was the $2^{\text {nd }}$ Trade Study. The maximum spin hoop stress and the spin hoop stress at the bore were used as gauges to compare disks with different processes. Though the stress values are similar for the baseline case (Figure 8) and Trade Study 1 (Figure 9), the hoop stress distribution is slightly different. Among the three cases, the best case is Trade Study 2; Ring oil quench with the lowest maximum spin hoop stress of $1175 \mathrm{MPa}$ and the lowest spin hoop stress at bore of 868 MPa (Figure 10).

$$
\begin{gathered}
\text { Pancake - oil quench } \\
\text { Baseline - Group } 2
\end{gathered}
$$
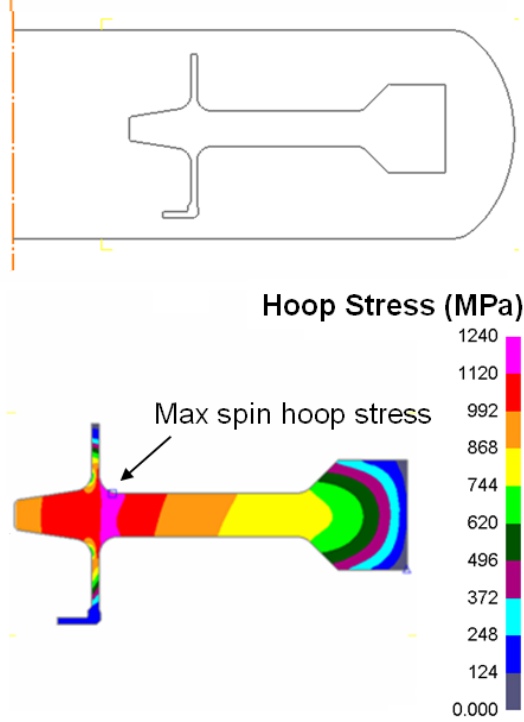

Figure 8. The baseline case for Trade Study: pancake oil quench with a maximum spin hoop stress of $1193 \mathrm{MPa}$ and a bore stress of $889 \mathrm{MPa}$. 


\section{Ring- water quench}

Trade study 1

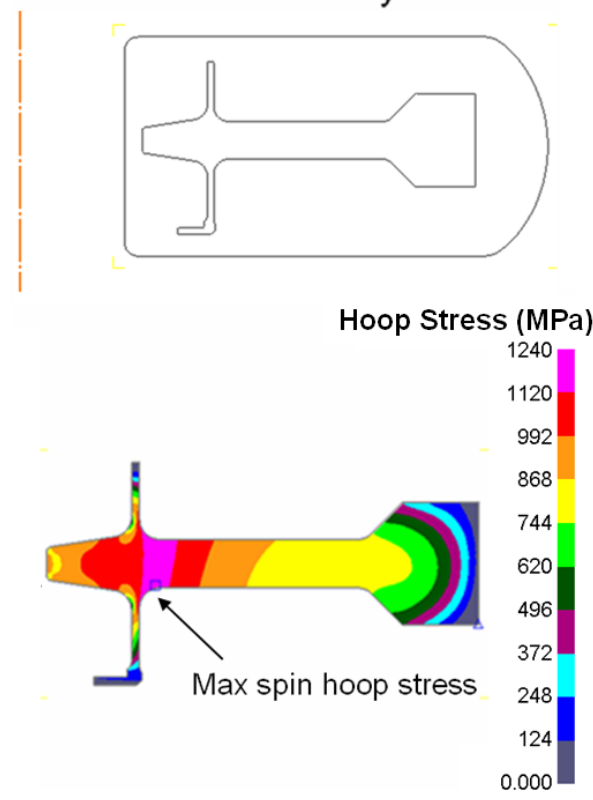

Figure 9. Trade Study 1: ring water quench with a maximum spin hoop stress of $1195 \mathrm{MPa}$ and a bore stress of $889 \mathrm{MPa}$.

\section{Ring - oil quench}

Trade study 2

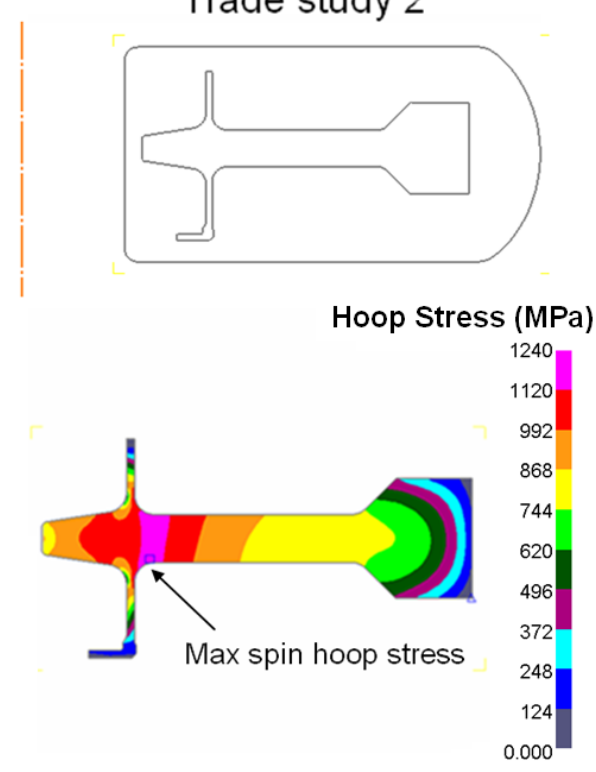

Figure 10. Trade Study 2: ring oil quench with a maximum spin hoop stress of $1175 \mathrm{MPa}$ and a bore stress of $868 \mathrm{MPa}$.
Maximization of component service life is related to minimization of the residual stress and service stress for critical locations. For a given heat treatment shape and pre-spin condition, the minimization of the residual stress and service stress is related to the optimization of the heat transfer coefficients (HTC's) during post solution cooling. This is Step 1 of the loop shown in Figure 7. The HTC segments for the 718 ring studied in this case are shown in Figure 11. Controlled cooling needs to be applied to realize a set of optimized HTC's. To optimize the HTC's, the optimization software iSight ${ }^{\mathrm{TM}}$ [8] was linked to the automated process modeling loop (Figure 7) to select HTC's. Figure 12 shows the iSight Design of Experiments (DOE) used to determine the minimum residual and service stresses for locations of interest to maximize component life.

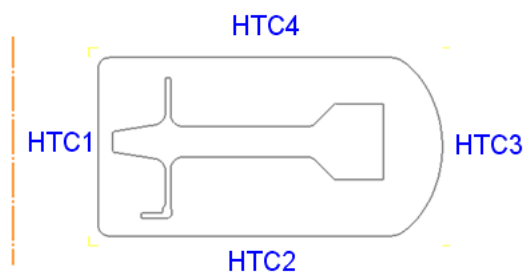

HTC (Heat Transfer Coefficient)

Figure 11. The HTC segments used for the ring of alloy 718 studied in this case.

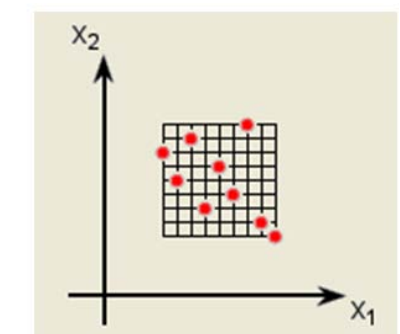

a)

b)

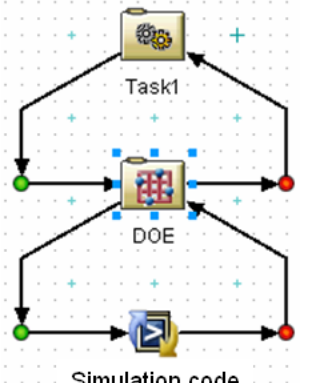

Simulation code
Figure 12. Schematics of Design of Experiments in iSight: a) Latin Hypercube DOE was used; b) DOE controls the simulation code which is the automated process modeling loop shown in Figure 7.

Thirty DOE trials were run in iSight for the disk with and without pre-spin and the impact of HTC's on the maximum spin hoop stress was analyzed. The thirty cases with pre-spin were analyzed first. Figure 13 is a Pareto plot showing the impact of the HTC's on the maximum spin hoop stress for the disk with pre-spin. It is shown that HTC3 has the largest impact (\% effect) on the maximum spin hoop stress. The maximum spin hoop stress will increase with an increase in HTC3. Thus, the HTC3 should be reduced if possible. Figure 13 also shows that the HTC1 has the next largest impact on the maximum spin hoop stress. The maximum spin hoop stress will be reduced if HTC1 increases. Thus, HTC 1 should be increased if possible. HTC2 and HTC4 have a minor impact on the maximum spin hoop stress and need 
to be increased in order to reduce the maximum spin hoop stress as well. Figure 14 shows the DOE results, the maximum spin hoop stress, for all HTC combinations as well as the potential optimum combination. The potential optimum combination of HTC1-HTC4 and the resultant spin hoop stress is shown in Figure 15. The maximum spin hoop stress is $1080 \mathrm{MPa}$ and the spin hoop stress at bore is $716 \mathrm{MPa}$. Both stresses are the lowest so far with a pre-spin condition (Group 2, Trade Study 1, and Trade Study 2).

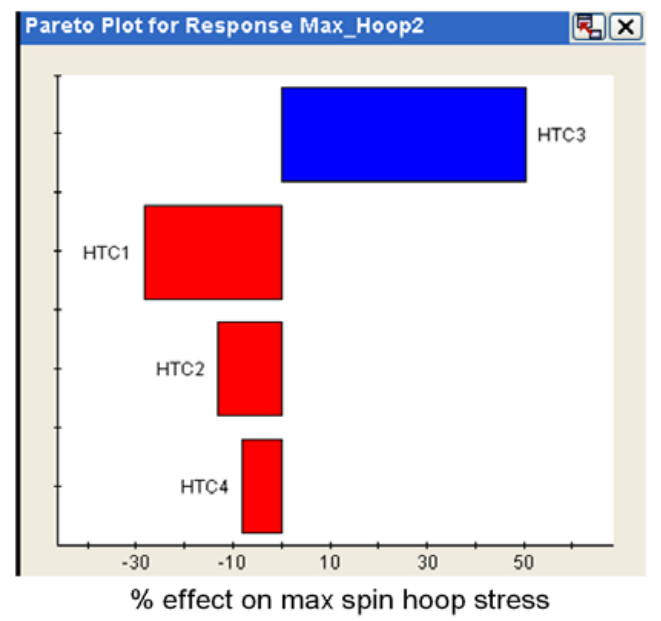

Figure 13. Pareto plot showing HTC's impact on max spin hoop stress with pre-spin.

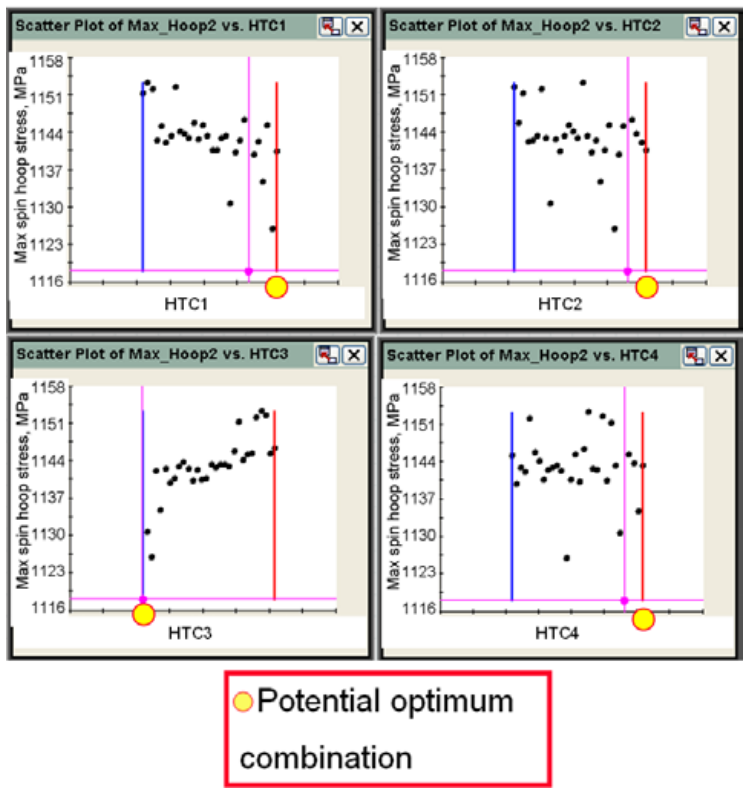

Figure 14. Individual HTC settings for HTC1-HTC4 in thirty trial DOE and the corresponding maximum spin hoop stress. The potential optimum combination of HTC1-HTC4 is also marked.

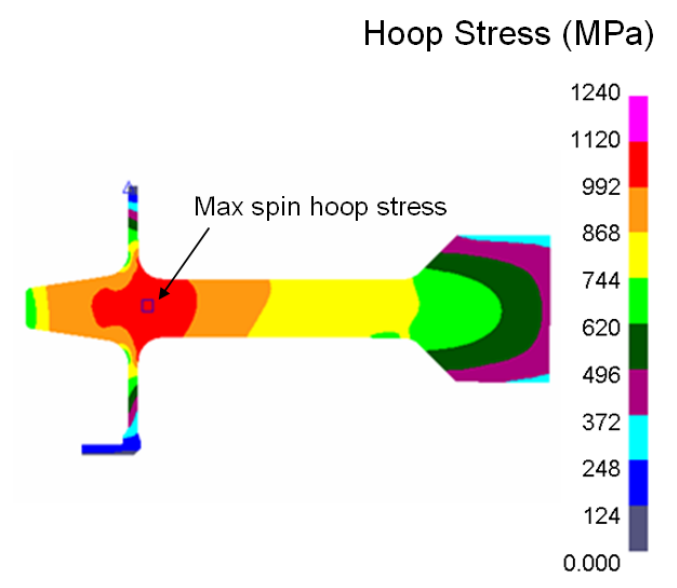

Figure 15. The potential optimum combination of HTC1-HTC4 setting for a superalloy 718 disk with pre-spin. The maximum spin hoop stress is $1080 \mathrm{MPa}$ and the bore stress is $716 \mathrm{MPa}$.

Although pre-spinning can reduce the spin hoop stress, there is extra cost and risk involved. It would be more desirable to obtain a similar stress state without pre-spinning the disk. Controlled cooling can be used rather than pre-spinning to yield the bore and create a compressive stress. The impact of HTC's on the maximum spin hoop stress for thirty DOE trials on the disk without pre-spinning were analyzed below. Figure 16 is a Pareto plot of the impact (\% effect) HTC's have on the maximum spin hoop stress for a disk without pre-spinning. It is shown that the HTC1 has the largest impact on reducing the maximum spin hoop stress. Thus, efforts should be made to increase HTC1 within manufacturing limit to reduce the maximum spin hoop stress. Figure 16 shows HTC2 and HTC4 again have a minor impact on the maximum spin hoop stress and need to be increased as well. An increase in HTC3 will increase the maximum spin hoop stress; however HTC3 has the least impact on maximum spin hoop stress. Figure 17 shows the DOE results, the maximum spin hoop stress, for all HTC combinations as well as the potential optimum combination.

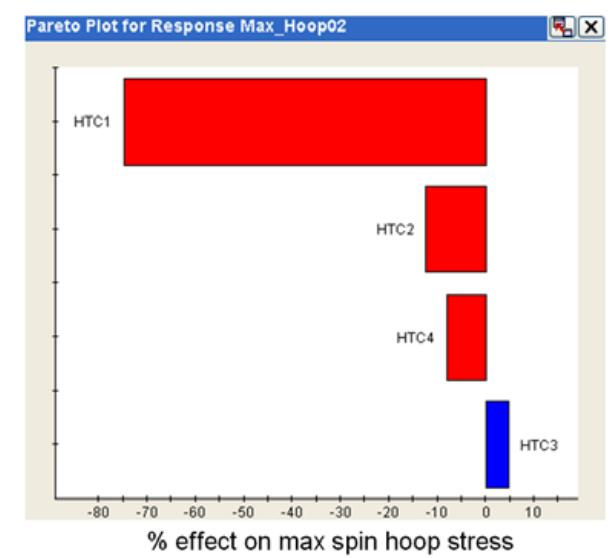

Figure 16. Pareto plot showing HTC’s impact on max spin hoop stress without pre-spinning. 
The potential optimum combination of HTC1-HTC4 setting was used in another modeling loop run and the spin hoop stress is shown in Figure 18. The figure shows that, even without prespinning, the maximum spin hoop stress is $1082 \mathrm{MPa}$ and the spin hoop stress at bore is $717 \mathrm{MPa}$. Both values are almost identical to the pre-spun disk shown in Figure 15. Thus, if the controlled cooling is performed properly, similar stress level could be achieved for the superalloy 718 disk with or without pre-spin. Obviously, the non pre-spun 718 disk is a more economical solution; it removes a process step and a potential risk.

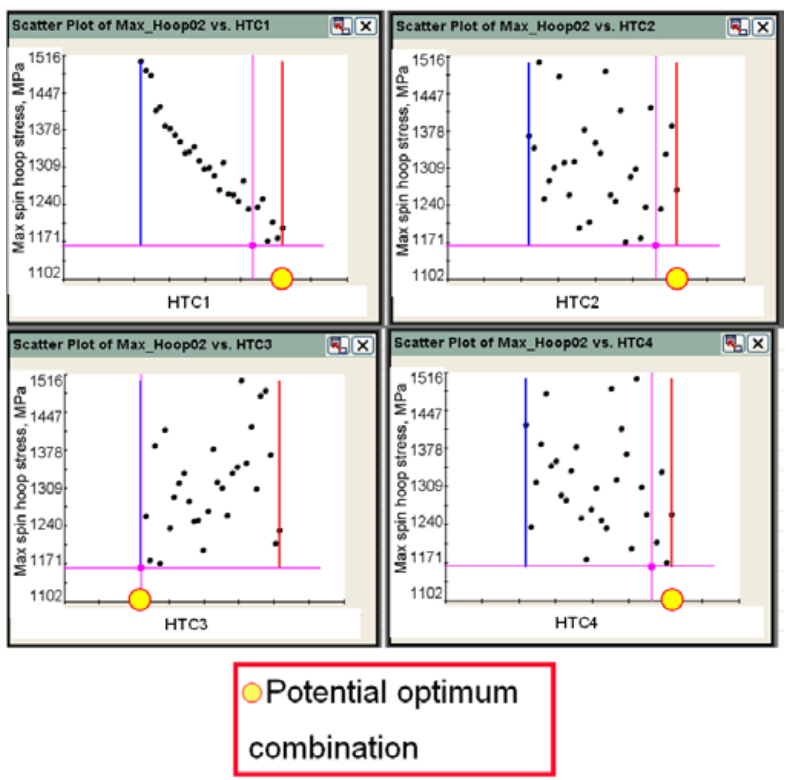

Figure 17. Individual HTC settings of thirty DOE without prespinning and the corresponding maximum spin hoop stress. The potential optimum combination is marked.

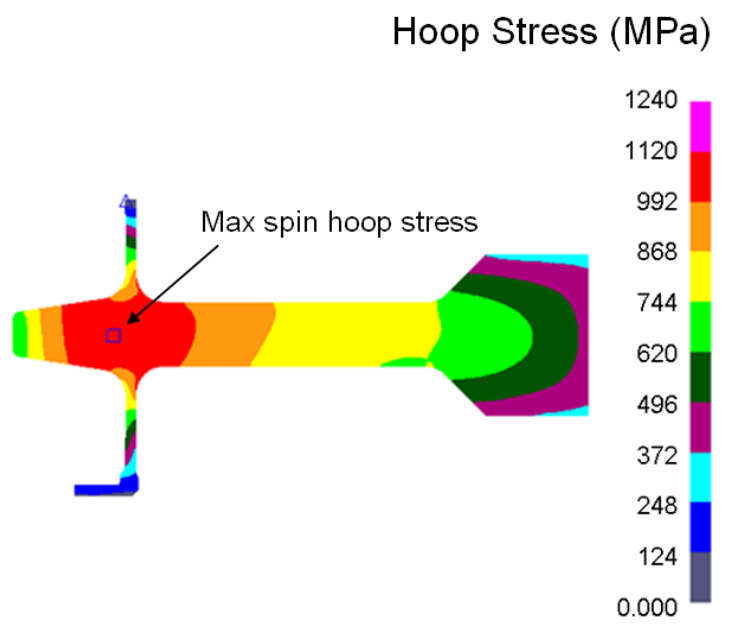

Figure 18. The potential optimum combination setting for the superalloy 718 disk without pre-spinning. The maximum spin hoop stress is $1082 \mathrm{MPa}$ and the bore stress is $717 \mathrm{MPa}$.

\section{Conclusions}

The residual stress distribution from the heat treatment processes plays a very important role in determining the service stress for locations critical to the life of rotating superalloy components. The residual stress modeling tool is maturing toward accurate prediction of residual stresses in final components. A spin stress prediction that included residual stress is consistent with the observed fracture pattern and the observed life for the superalloy 718 disks presented in this paper. The automated residual stress/service stress/lifing calculation loop makes the optimization of component design more efficient and practical for use within aggressive design timescales. Controlled cooling could be used to maximize the disk performance with possible lower cost and is the future for residual stress/service stress/lifing optimization.

\section{Acknowledgements}

The authors would like to acknowledge the support by colleagues at Rolls-Royce for this work. Thanks go to Dr. Mark Hardy, Mr. Ken Green and Dr. Randy Helmink at Rolls-Royce and to Eric Huron at GE for their editing and comments on this paper.

(C) Copyright Rolls-Royce Corporation 2012

\section{References}

1. Committee on Integrated Computational Materials Engineering,National Research Council, "Integrated Computational Materials Engineering: A Transformational Discipline for Improved Competitiveness and National Security", The National Academies Press, Washington, D.C, 2008.

2. R. A. Wallis, "Modeling of Quenching, Residual Stress Formation, and Quench Crack,” Ed. D. U. Furrer, and L. S. Semiatin, Metal Process Simulation, ASM Handbook, ASM International, Vol. 22B, 2010, pp. 547-585.

3. R. A. Wallis, N. M. Bhathena, P. R. Bhowal, and E. L. Raymond, "The Application of Process Modeling to Heat Treatment of Superalloys," Industrial Heating, Vol. LV, No. 1, 1988, pp. 30-33.

4. J. M. Franchet, F. Devy, P. E. Mosser, Y. Honnorat, and A. Benallal, "Modeling during the Oil Quenching of an Astroloy Turbine Disk," Superalloys 1992, TMS. Warrendale, PA, 1992, pp. 73-82.

5. D. U. Furrer, R. Shankar, and C. White, "Optimizing the Heat Treatment of Ni-Based Superalloy Turbine Discs," JOM, March 2003, pp. 32-34.

6. DEFORM, trademark of Scientific Forming Technologies Corporation, Columbus, $\mathrm{OH}$.

7. M. G. Glavicic, R. L. Goetz, G. Shen, J. A. Rasche, R. A. Ress III, and J. Stillinger, "Application of ICME to Turbine Engine Component Design Optimization,” 52th AIAA/ASME/ASCE/AHS/ASC Structures, Structural Dynamics, and Materials Conference, Denver, Colorado, April, 2011.

8. iSight, trademark of Dassault Systems. 